\title{
Investigation on the Effect of Quantitative Self-Rating Health Scale in Elderly Patients with Hypertension
}

Guangjie Li, Qixin Du', Xuanxuan Wang ${ }^{2,3}$, HUA LI, JIAO CHEN ${ }^{4}$, GUO LU ${ }^{4}$ Hongping Cheng ${ }^{5}$, Xun Gong ${ }^{6 *}$ AND XIAOWEI $\mathrm{WU}^{7 *}$ 
Medical Administration Division of Cadre sanatorium of Hainan \& Geriatric hospital of Hainan (CSH), Haikou, ${ }^{1} \mathrm{Nursing}$ Department of Cadre sanatorium of Hainan \& Geriatric hospital of Hainan (CSH), Haikou, ${ }^{2}$ Department of Integrated TCM \& Western Medicine, Hubei Cancer Hospital, Wuhan, Hubei, ${ }^{3} \mathrm{Clinical}$ College of Chinese Medicine, Hubei University of Chinese Medicine, Wuhan, Hubei, ${ }^{4}$ School of International Education, Hainan Medical University, Haikou, ${ }^{5}$ School of public health, Hubei Medical College, Shiyan,Hubei, ${ }^{6}$ Center for civil-military inosculation and development of new material industry in Huludao City, Huludao, ${ }^{7}$ Department of Thoracic Surgery, TongJi Hospital, TongJi Medical College, Huazhong University of Science and Technology, Wuhan, 430030, China *Co-authors:Guangjie Li, Qixin Du contirbute same to this work. \#Corresponding Author: Xun Gong, Xiaowei Wu

\section{II et al.: Effect of Self-Rating Health Scale in Patients with Hypertension}

To investigate and analyze the effect of quantitative self-rating health scale in elderly patients with hypertension. From January 2018 to October 2018, 95 elderly hypertensive patients managed by a community health service center in a city were selected, and 100 healthy persons were selected in the same period. Self-rated health assessment scale and general data questionnaire were used to investigate and analyze them. The total score of self-rated health assessment scale and its dimensions in elderly patients with hypertension were significantly lower than those in healthy control group $(p<0.05)$, except that there was no significant difference in cognitive function $(p>0.05)$. The scores of physical health, social health and self-rated health assessment scale in elderly patients with hypertension were correlated with gender, age and educational level in varying degrees $(p<0.05)$, but not with marital status and living conditions $(p>0.05)$. The scores of mental health and total self-rated health were correlated with age and educational level $(p<0.05)$, but not with gender, marital status and living condition $(p>0.05)$. Multiple stepwise regression analysis showed that age was an important factor affecting self-rated health assessment scale in elderly patients with hypertension $(\mathbf{p}<0.05)$. The sex, age and education are closely related to self-rated health assessment scale scores. Effective measures should be taken to improve health status and quality of life of elderly patients with hypertensions.

Key words: Self-rated health Scale, elderly patients, hypertension

Now-a-days, China has entered into an aging society, and the health status of the elderly has been increasingly concerned by the society. Hypertension is one of the main risk factors for the occurrence of cardiovascular and cerebrovascular diseases, which seriously threatens the health of the elderly ${ }^{[1,2]}$. The occurrence mechanism of hypertension is related to many factors, among which psychosocial factors play a certain role in its occurrence and development, and can cause the fluctuation of blood pressure. Therefore, to explore the influence of psychosocial factors on the occurrence of hypertension is of great significance for the prevention and treatment of this disease. Self-assessment of health is a subjective assessment of people's health status, including physical, psychological and social health, which is closely related to the quality of an individual's life ${ }^{[3,4]}$. In order to understand the self-rated health status and related factors of elderly patients with hypertension, this paper investigated the self-rated health status of 95 elderly patients with hypertension, and discussed the influence of physiological, psychological and social factors on the occurrence and development of hypertension, so as to provide reference for improving the quality of life of patients. The report is as follows. A stratified, random

*Address for correspondence

E-mail: rongweiwo108@163.com sampling method was adopted to select 95 elderly patients with hypertension who were managed by a community health service center in a city from January 2018 to October 2018. Inclusion criteria: Age \& GT; The age of 60; Meet the relevant diagnostic criteria of hypertension: non-drug systolic blood pressure (SBP) $\geq 140 \mathrm{~mm} \mathrm{Hg}$ and (or) diastolic blood pressure (DBP) $\geq 90 \mathrm{~mm} \mathrm{Hg}$; The consciousness is clear, the life can take care of oneself person, volunteer to join investigator. Exclusion criteria: Patients with secondary hypertension; Consciousness disorder or severe liver and kidney dysfunction; those with severe acute or chronic diseases. A total of 100 healthy subjects were included in the healthy control group. The table, designed by the researchers themselves, mainly covers age, sex, education, marital status and residence status. Among the elderly patients with hypertension, 55 were male and 40 were female. The average age was

This is an open access article distributed under the terms of the Creative Commons Attribution-NonCommercial-ShareAlike 3.0 License, which allows others to remix, tweak, and build upon the work non-commercially, as long as the author is credited and the new creations are licensed under the identical terms

Accepted 28 December 2020

Revised 28 November 2020 Received 25 May 2020 Indian J Pharm Sci 2020;82(6):1062-1066 
$(64.31 \pm 10.52)$ between 60 and 87 y old. Education level: 25 cases in primary school, 35 cases in junior middle school, 24 cases in senior high school and technical secondary school and 11 cases in university. Marital status: 64 cases were married, 21 cases were widowed, and the other 10 cases. Living conditions: 15 cases lived alone, 48 cases lived with spouse and 32 cases lived with children. Among the 100 healthy patients, there were 58 males and 42 females. The average age was $(63.83 \pm 10.34)$ y old. Education: 28 cases in primary school, 36 cases in junior middle school, 26 cases in senior high school and technical secondary school, and 10 cases in university. Marital status: 67 cases were married, 22 cases were widowed, and the other 11 cases. Living conditions: 18 cases lived alone, 49 cases lived with spouse and 33 cases lived with children. There was no significant difference between the two groups in terms of age, gender, educational level and other general information (P\&gt;0.05). The quantitative self-rated health scale designed by Xu Jun in China was adopted ${ }^{[5]}$. The scale includes 10 dimensions, a total of 48 item, including physiological, psychological and social health three child scale, and includes three dimensions of each scale, self-reported physical health sub scale including 1 17 items, since the reason health scale including 19 to 33 items, self-test social health sub scale including 35 46 items, overall self-test health including 18, 34, 47, 48 entries, become the tenth dimension. The score of this scale adopts the simulated linear method. The highest theoretical score of each item is 10 , and the lowest score is 0 . Subjects were included in strict accordance with the inclusion and exclusion criteria; All surveyors received centralized training, unified instruction language and unified professional training; Obtain the informed consent and cooperation of the research subjects, inform them of the purpose and significance of the survey, and answer questions timely in the process of issuing the questionnaire. The questionnaire shall be anonymously distributed and collected on site. Review the completed questionnaire and add missing indicators. SPSS 22.0 statistical software was used to process the data, and the count data was represented by $\mathrm{N}(\%)$. Comparison between groups was performed by chi-square test, while measurement data was represented by (). Comparison between groups was performed by T-test and Pearson correlation analysis was performed to analyze the correlation between SRHMS dimension indicators and demographic indicators, and a multiple linear regression analysis was performed, P\&lt. A difference of 0.05 was statistically significant. As shown in Table 1, SRHMS scores and all dimension scores of elderly patients with hypertension were significantly lower than those of healthy controls (P\&lt; 0.05). The physiological health scores of elderly patients with hypertension were correlated with gender, age and educational level to different degrees (P\&lt;0.05), had no relation with marital status and residential status $(\mathrm{p}>0.05)$.Patients' mental health scores were correlated with age and educational level to varying degrees (P\&lt; $(p>0.05)$ was not correlated with gender, marital status and residential status ( $p>0.05)$.Patients' social health scores were correlated with gender, age, and educational level to varying degrees (P\&lt;0.05), had no relation with marital status and residential status ( $\mathrm{p}>0.05)$.Patients' overall selfmeasured health scores were correlated with age and educational level to different degrees (P\&lt; $(\mathrm{p}>0.05)$ was not correlated with gender, marital status and residential status $(\mathrm{p}>0.05)$.SRHMS scores were

\section{TABLE 1: COMPARISON OF SRHMS STATUS BETWEEN THE TWO GROUPS OF ELDERLY}

\begin{tabular}{|c|c|c|c|c|c|}
\hline Project & $\begin{array}{l}\text { Total } \\
\text { score }\end{array}$ & $\begin{array}{l}\text { High blood pressure } \\
\text { group }(n=95)\end{array}$ & $\begin{array}{l}\text { Healthy control } \\
\text { group }(n=100)\end{array}$ & T value & $P$ values \\
\hline Physiological health sub-scale & 170 & $137.69 \pm 11.45$ & $153.63 \pm 10.78$ & 10.013 & $<0.001$ \\
\hline Somatic symptoms and organ function & 70 & $52.08 \pm 6.41$ & $58.81 \pm 6.90$ & 7.047 & $<0.001$ \\
\hline Daily life function & 50 & $48.36 \pm 1.52$ & $49.05 \pm 1.41$ & 3.288 & 0.001 \\
\hline Physical function & 50 & $37.25 \pm 7.49$ & $45.77 \pm 7.63$ & 7.864 & $<0.001$ \\
\hline Mental health subscale & 150 & $115.31 \pm 11.46$ & $124.80 \pm 12.52$ & 5.513 & $<0.001$ \\
\hline Positive emotions & 50 & $39.05 \pm 5.36$ & $44.12 \pm 6.50$ & 5.926 & $<0.001$ \\
\hline Psychological symptoms and negative emotions & 70 & $55.85 \pm 6.14$ & $59.12 \pm 5.53$ & 3.912 & $<0.001$ \\
\hline Cognitive function & 30 & $20.41 \pm 2.69$ & $21.16 \pm 3.42$ & 1.696 & 0.091 \\
\hline Social health subscale & 120 & $86.89 \pm 11.59$ & $96.47 \pm 10.54$ & 6.044 & $<0.001$ \\
\hline Role activities and social adaptation & 40 & $33.04 \pm 1.85$ & $37.12 \pm 1.52$ & 16.863 & $<0.001$ \\
\hline Social support & 30 & $20.01 \pm 3.57$ & $24.21 \pm 2.36$ & 9.737 & $<0.001$ \\
\hline Overall self-reported health & 40 & $31.45 \pm 3.45$ & $35.41 \pm 2.50$ & 9.212 & $<0.001$ \\
\hline Total score & 440 & $371.34 \pm 28.48$ & $410.31 \pm 25.45$ & 10.086 & $<0.001$ \\
\hline
\end{tabular}

**. The correlation was significant when the confidence (double measure) was $0.01,{ }^{*}$. The correlation was significant when the confidence (double measure) was 0.05 
correlated with gender, age, and educational attainment to varying degrees (P\&lt;0.05), had no relation with marital status and residential status $(\mathrm{p}>0.05)$ are shown in Table 2. With gender $(\mathrm{m}=1, \mathrm{f}=2)$, age (y old), degree of culture (elementary school $=1=2$, junior high school, high school and technical secondary school $=3=4$, university), marital status (married $=1$, widowed $=2$, other $=3$ ) and living conditions (=who live alone, living with a spouse $=2,1$ with children $=3$ ) as the independent variable, in elderly patients with high blood pressure SRHMS total score as the dependent variable, multiple stepwise regression analysis results showed that age is the important factors that affect the old patients with hypertension SRHMS (P\&lt;0.05) (Table 3). With the change of modern health concept, people's health assessment is not only limited to physical diseases, but also comprehensive assessment from psychological, social and other perspectives. Suchma in the $1950 \mathrm{~s}$, such as the first in this paper, the definition of selfevaluation of health, through constant practice and improvement, now SRHMS scale has become a common international recognition of self-health assessment tool, which include physical health, mental health, social health, it is relatively comprehensive and accurately reflect the health of people, is a complement to the measurement approach to traditional health ${ }^{[6,7]}$. Domestic scholars have also conducted in-depth studies on the physical and mental health conditions of different groups ${ }^{[8,9]}$. At present, there is no radical cure for hypertension, which can only be controlled within a certain range by means of antihypertensive drugs and life intervention. However, long-term use of drugs and long-term anxiety and tension will seriously affect the quality of life of patients ${ }^{[10,11]}$. This study, the survey found in elderly patients with hypertension physiological health sub scale scores, physical symptom and organ function, the functions of daily life, physical activity, mental health scale score, positive emotion, mood, psychological symptoms and the negative social health sub scale scores, role activity and social adaptation, social resource and social contact, social support and overall self-test health scores, total scores of SRHMS were lower than the healthy control group, difference was statistically significant, and there were no significant differences in cognitive function $(\mathrm{p}>0.05)$. It is suggested that the mental health problems of elderly patients with hypertension are prominent, and attention should be paid to the physical and mental health of the patients, and scientific and effective psychological intervention measures should be taken actively to improve the quality of life of the patients. The total score of SRHMS was related to gender, age and education level of patients. Multiple factors, according to the results of correlation analysis of senile hypertension patients' physical health score, social health, mental health score points, overall self-test

TABLE 2: CORRELATION ANALYSIS BETWEEN SRHMS AND VARIOUS FACTORS IN ELDERLY PATIENTS WITH HYPERTENSION (R)

\begin{tabular}{|c|c|c|c|c|c|c|}
\hline & & Gender & Age & $\begin{array}{l}\text { Level of } \\
\text { education }\end{array}$ & $\begin{array}{l}\text { Marital } \\
\text { status }\end{array}$ & $\begin{array}{c}\text { Living } \\
\text { conditions }\end{array}$ \\
\hline \multirow{2}{*}{$\begin{array}{l}\text { Physiological health sub- } \\
\text { scale }\end{array}$} & Pearson The correlation & $-0.207^{*}$ & $-0.633^{* *}$ & $0.270^{* *}$ & -0.125 & -0.158 \\
\hline & Significance (double tails) & 0.044 & $<0.001$ & 0.008 & 0.229 & 0.126 \\
\hline \multirow{2}{*}{ Mental health subscale } & Pearson The correlation & -0.197 & $-0.643^{* *}$ & $0.261^{*}$ & -0.117 & -0.167 \\
\hline & Significance (double tails) & 0.056 & $<0.001$ & 0.011 & 0.258 & 0.105 \\
\hline \multirow{2}{*}{ Social health subscale } & Pearson The correlation & $-0.211^{*}$ & $-0.598^{* *}$ & $0.259^{*}$ & -0.117 & -0.148 \\
\hline & Significance (double tails) & 0.040 & $<0.001$ & 0.011 & 0.259 & 0.153 \\
\hline \multirow{2}{*}{$\begin{array}{l}\text { Overall self-reported } \\
\text { health }\end{array}$} & Pearson The correlation & -0.191 & $-0.612^{* *}$ & $0.271^{* *}$ & -0.110 & -0.153 \\
\hline & Significance (double tails) & 0.063 & $<0.001$ & 0.008 & 0.287 & 0.139 \\
\hline \multirow{2}{*}{ Total score } & Pearson The correlation & $-0.204^{*}$ & $-0.624^{* *}$ & $0.264^{* *}$ & -0.119 & -0.157 \\
\hline & Significance (double tails) & 0.047 & $<0.001$ & 0.010 & 0.251 & 0.128 \\
\hline
\end{tabular}

${ }^{* *}$. The correlation was significant when the confidence (double measure) was $0.01,{ }^{*}$. The correlation was significant when the confidence (double measure) was 0.05

TABLE 3: STEPWISE REGRESSION ANALYSIS OF SRHMS IN ELDERLY PATIENTS WITH HYPERTENSION

\begin{tabular}{|c|c|c|c|c|c|}
\hline & \multicolumn{2}{|c|}{ Nonstandardized coefficient } & \multicolumn{3}{|c|}{ Thestandardcoefficient } \\
\hline & B & The standard error & beta & $\mathrm{t}$ & significant \\
\hline (constant) & 526.871 & 22.825 & & 23.083 & $<0.001$ \\
\hline gender & -5.998 & 4.840 & -0.104 & -1.239 & 0.219 \\
\hline age & -1.979 & 0.303 & -0.572 & -6.523 & $<0.001$ \\
\hline Level of education & 2.616 & 2.526 & 0.089 & 1.036 & 0.303 \\
\hline Marital status & -3.763 & 3.528 & -0.089 & -1.067 & 0.289 \\
\hline Living conditions & -1.701 & 3.343 & -0.042 & -0.509 & 0.612 \\
\hline
\end{tabular}


health score and total score and age, culture level have different degrees of correlation, gender and physical health scale, social health scale and total score with different degree of correlation, the scale has nothing to do with the marital status and living conditions, the related scholars also had similar conclusion ${ }^{[12,13]}$. Sun Kaige et al. found that the overall health literacy and various literacy of hypertension patients were positively correlated with their educational level and negatively correlated with their age ${ }^{[14]}$. On the one hand, with the increase of age, physical function gradually decline, plus cognitive and learning ability decline, the ability to acquire knowledge decreased; On the other hand, those with higher education level are better than those with lower education level in reading level, understanding level, sense of self-health care, ability to obtain and use information and utilization of social resources. The results of multiple stepwise regression analysis showed that age was an important factor affecting SRHMS in elderly patients with hypertension, and gender and educational level were not included in the model. Tip should strengthen the health management on hypertension in aged patients, clinical psychological intervention measures, adjust the negative mood, cultivating their proper exercise consciousness, improve the body symptoms, to establish a healthy lifestyle, make full use of social resources, to maintain the harmonious interpersonal relationship, maintain a healthy society and improve the patient's physiological, psychological and social function ${ }^{[15-16]}$. For the managers of community health service centers, elderly patients should be helped to obtain health-related information, actively publicize health knowledge related to chronic diseases, have regular free physical examinations, and promote healthy behaviors to gradually improve their health status ${ }^{[17]}$. Considering that this study is a crosssectional study, there is no long-term follow-up observation of the respondents and no dynamic analysis of the impact of relevant factors on the physical, mental and social health of elderly patients with hypertension, which needs to be further discussed.

\section{REFERENCES}

1. Juyang X, Xuetao T, Kun D. Influence of community intervention on blood pressure control effect of hypertension patients in Guiyang. Med Soc 2014;27:10-3.
2. Fang L, Yan G, Liang W. Current situation and influencing factors of blood pressure control in community patients with hypertension. Public Health Prevent Med 2014;25:100-2.

3. Zhu L, Zhu G, Meng M. Analysis of bengbu residents' selfrated health status and its influencing factors. Chin J Dis Control 2018;22:91-4.

4. Jiehua L, Yue L, Bing Z. Empirical Analysis of the interaction between social participation and self-rated health of the elderly in Mainland China- Based on CLHLS data test. Population research 2017;41:17-28.

5. Jun X, Binhui W, Minyan H. Development and evaluation of self-test Health Rating Scale. Chin J Behavioral Med 2000;9:65-8.

6. Xiaodan W, Jiehua L. Empirical Analysis on influencing Factors of seasonal Migratory Bird elderly self-evaluation on health- A Case study of Hainan Province. Beijing Soc Sci 2017;32:101-9.

7. Lin L, Shan H, Ying Y. Capacity-stress model study on elderly self-assessment of health- A Case study of Guangzhou community. Geograph Sci 2018;38:548-56.

8. Huiwen T, Xiao Z. Comparison of urban and rural elderly selfrated health status based on Fairlie decomposition method. Public Health Chin 2018;34:55-9.

9. Wei Z, Xiao H, Chunjiao Y. Study on the use of antihypertensive drugs and the status quo of blood pressure control in urban and rural patients with hypertension in Jiangxi Province. Chin J General Pract 2018;21:90-6.

10. Sha W, Honghua Y, Xiuhua W. Research on the influence of a new community chronic disease team management model led by community nurses on self-efficacy and compliance of hypertension patients. Chin J General Pract 2017;20:3343-8.

11. Mcnaughton CD, Jacobson TA, Kripalani S. Low literacy is associated with uncontrolled blood pressure in primary care patients with hypertension and heart disease. Patient Edu Counsel 2014;96:165-70.

12. Perez A. Acculturation, Health Literacy, and Illness Perceptions of Hypertension among Hispanic Adults. J Transcult Nurs 2014;26.

13. Huihui Y, Zhen L, Shukang W. Adverse mood and related factors in elderly patients with hypertension and diabetes mellitus. J Shandong University Med Sci 2015;53:81-5.

14. Kaige S, Shiyan W, Kang H. Current status of health literacy of hypertensive patients in the suburbs of Beijing and its correlation with blood pressure control. Health Edu Chin 2017;33:684-9.

15. Shenhong G, Yanling S, Yunyun L. Analysis on the application status of general practice health management in community patients with hypertension. Chin General Pract 2017;20:236-7.

16. Qing Z, Yiling T. Implementation effect analysis of the management project for the elderly and chronic disease patients in public and private community health service institutions in Haikou city. Modern Prevent Med 2017;44:88-91.

17. Qiangwei F, Xunan W, Linsheng Y. Research on depressive symptoms and its correlation with chronic diseases among the elderly in urban and rural communities of $\mathrm{Lu}$ 'an city. Modern Prevent Med 2018;45:102-105. 